\title{
Disorder classification of the vibrational spectra of modern glasses
}

\author{
Zhiwen Pan ${ }^{1}$, Omar Benzine ${ }^{1}$, Shigeki Sawamura ${ }^{2}$, Rene Limbach ${ }^{1}$, Akio Koike $^{2}$, \\ Thomas D. Bennett ${ }^{3}$, Gerhard Wilde ${ }^{4}$, Walter Schirmacher ${ }^{5}$, and Lothar Wondraczek ${ }^{1}$ \\ ${ }^{1}$ Otto Schott Institute of Materials Research, University of Jena, 07743 Jena, Germany \\ ${ }^{2}$ Materials Integration Laboratories, AGC Inc., Yokohama, Kanagawa 230-0045 Japan \\ ${ }^{3}$ Department of Materials Science and Metallurgy, University of Cambridge, \\ Charles Babbage Road, Cambridge, CB3 OFS, United Kingdom \\ ${ }^{4}$ Institute of Materials Physics, University of Münster, 48149 Münster, Germany and \\ ${ }^{5}$ Institute of Physics, University of Mainz, 55099 Mainz, Germany
}

\begin{abstract}
Using the coherent-potential approximation in heterogeneous-elasticity theory with a log-normal distribution of elastic constants for the description of the Raman spectrum and the temperature dependence of the specific heat, we are able to reconstruct the vibrational density of states and characteristic descriptors of the elastic heterogeneity of a wide range of glassy materials. These descriptors are the non-affine contribution to the shear modulus, the mean-square fluctuation of the local elasticity, and its correlation length. They enable a physical classification scheme for disorder in modern, industrially relevant glass materials.

We apply our procedure to a broad range of real-world glass compositions, including metallic, oxide, chalcogenide, hybrid and polymer glasses. Universal relationships between the descriptors on the one side, and the height and frequency position of the boson peak, the Poisson ratio and the liquid fragility index on the other side are established.
\end{abstract}

In contrast to crystalline materials, glasses exhibit aperiodic heterogeneity in local density, chemical composition and structural rigidity. This complicates the extraction of structureproperty correlations and predictive tools for experimental materials design or classification. On the other hand, statistical information may be obtained from the vibrational spectrum of the materials, which can be probed by Raman light scattering and by measuring the temperature dependence of the specific heat. Here we use a meanfield theory, which links the vibrational spectrum to the statistics of elastic heterogeneity, to characterize glasses according to their disorder strength and the characteristic correlation length of spatial elastic fluctuations. These descriptors are shown to be related to the elastic properties of the glass and the temperature dependence of the viscosity of the melt ("glass fragility") and may therefore be helpful for designing new materials for specific applications.

The extraordinary spatial homogeneity of glassy materials underlies their many applications, as it leads to, e.g., optical transparency, characteristic fracture patterns, distinct mechanisms of charge, heat and sound transport, or specific solvation behavior. In turn, the ultimate limit of all of these properties lies in the length scale below which material homogeneity is broken. Following the curious observation that the density of a glass is usually lower than that of its crystalline counterpart (when chemical compositions were equivalent) early hypotheses of network formation [1] and excess free volume
$[2,3]$ were formulated for different classes of glass. These led to the qualitative paradigm of glass structure, where aperiodic heterogeneity in the atomic packing density, in network rigidity and/or in the distribution of chemical species constitutes the primary difference to a crystalline lattice. Although the process by which a supercooled liquid acquires the rigid glassy state remains poorly understood $[4,5]$, such types of structural heterogeneity have been related to the heterogeneous dynamics of the liquid [6], liquid fragility [7] and the non-ergodicity factor [8].

However, across all types of glass, quantitative descriptors for structural heterogeneity have been elusive, due to the inability to describe glass structure beyond short length scales. As a result of structural disorder, the fundamental tool for understanding crystal properties, namely the lattice symmetry, is not available for glasses. Instead, empirical or semi-empirical regression models are frequently used for property predictions. For example, the elasticity of multi-component glasses is often described through mean-field regression analysis of bond energy density $[9,10]$. Although useful in their simplicity, such practical approaches ignore localization phenomena and non-affinity, which are common to disordered materials across the broadest range of length scales [11] and material chemistries [12]. For example, for brittle glasses with strongly covalent bonding, heterogeneous network topology has been identified to mediate nanoscale ductility [13] and macroscopic plasticity [14, 15].

It turned out that an important property of glasses is the presence of random spatial fluctuations of local elastic constants. It was shown that such heterogeneous elasticity is intimately related to the orrurrence of non-affine displacements [16-20]. In metallic glasses, a parameter 
denoted flexibility volume was extracted from the spatial distribution of the atomic mean-square displacement as probed by computational simulation [21]. For twoand three-dimensional Lennard-Jones glasses, correlated particle displacements with a correlation length of 20-30 interparticle spacings were found $[18,19]$. Similar observations were made in a computer-generated model of amorphous silica [19].

When compared to crystals, the vibrational density of states (VDoS) of glasses exhibits an anomalous excess in the $\mathrm{THz}$ regime, which forms a maximum if the VDoS is divided by the square of the frequency ("boson peak") [22-24]. The VDoS is directly accessible by means of inelastic nuclear [25] and incoherent neutron scattering [26], but only indirectly by inelastic coherent neutron [23, 24, 27], X-ray [28-30], and Raman [14, 31-33] scattering. Further information on the VDoS in the THz regime can be obtained from the temperature $(T)$ dependence of the specific heat $C(T)[34,35]$, where the boson peak is observed if plotted as $C(T) / T^{3}$. The possible origin of the boson peak has been discussed rather controversely [36-40]. However, many groups nowadays agree that in most cases spatial fluctuations of the local elastic moduli cause this anomaly [41-43], hence, studies of the $\mathrm{THz}$ vibrational anomaly in the VDoS - in principle - provide access to the descriptors of spatial elastic fluctuations.

The boson peak related anomalies, including a characteristic shoulder in the temperature dependence of the thermal conductivity, can be rather successfully explained and described by heterogeneous-elasticity theory (HET, [44]). Combined with the self-consistent Born approximation (SCBA) a relation of the excess VDoS with the sound attenuation coefficient was established [45] and the results where tested in detail against extensive molecular-dynamics simulations [41]. In the latter study it was also shown that the decrease of the value of the shear modulus with respect to the average one is due to non-affine elasticity, and that HET accounts for this effect.

As HET describes microscopically the vibrational dynamics of a glass, all spectroscopic data can be expressed in terms of the wavenumber-dependent dynamic susceptibilities, which are obtained from the statistics of the fluctuating elastic constants. In particular the Raman spectra are given as wavenumber integrals of these susceptibilities [31]. Therefore, by means of HET, it is possible to unambiguously extract the VDoS from Raman data $[31,33,46]$. This procedure has been demonstrated to be more reliable than using a phenomenological frequencydependent coupling constant $C(\omega)$, as is widely done in the literature $[14,31,32]$.

However, within SCBA the description of the elastic disorder is restricted to weak and Gaussian fluctuations. As this is not sufficient for the description of a very broad range of disordered materials, HET has been generalized by means of the coherent-potential approximation (CPA) $[36,47]$. Within CPA an arbitrary distribution of local elastic constants can be implemented, and there is no re- striction to weak disorder. However, a systematic analysis of a broad class of glasses by means of HET-CPA has not yet been conducted.

We now present the results of a disorder classification of modern glasses using HET-CPA with a log-normal distribution density of spatially fluctuating elastic constants. This variant of HET features specific descriptors of the statistics of the shear modulus, namely the variance, the geometric mean and the coarse-graining size, which is proportional to the correlation length of the fluctuations. Using the scaling properties of the CPA we obtain a relation between the first two quantities, which reduces the descriptors to the disorder parameter $\sigma^{2}$ and the coarse-graining wavenumber $k_{e}$. Evaluating the vibrational spectra of a large number of glassy materials we obtain correlations of these descriptors with the boson peak frequency, the boson peak height, the Poisson ratio and the liquid fragility index. We achieve this goal with experimental input from $\mathrm{THz}$ Raman spectroscopy and low-temperature heat capacity, covering all known classes of glass, from metallic to non-metallic, chalcogenide, polymeric and hybrid materials.

\section{Theory}

\section{HET-CPA}

Within HET-CPA the spatially fluctuating local shear moduli $G\left(\mathbf{r}_{i}\right) \equiv G_{i}$ are converted to a macroscopic complex, frequency-dependent modulus $G(z)(z=\omega+i \epsilon$, $\omega=2 \pi \nu$ is the angular frequency) by the self-consistent CPA equation

$$
\left\langle\frac{G_{i}-G(z)}{1+\frac{1}{3}\left[G_{i}-G(z)\right] \Lambda\left(k_{e}, z\right)}\right\rangle_{P}=0
$$

The (arithmetic) average $\langle\ldots\rangle_{P}$ is performed with a lognormal distribution density

$$
P\left(G_{i}, G_{0}, \sigma\right)=\frac{1}{\sigma \sqrt{2 \pi}} \frac{1}{G_{i}} \exp \left\{-\frac{1}{2 \sigma^{2}}\left[\ln \left(G_{i} / G_{0}\right)\right]^{2}\right\}
$$

We chose this distribution, because for weak disorder $\sigma^{2} \ll 1$ it reduces to a Gaussian distribution (see Fig. $1)^{1}$, but for $\sigma^{2}$ values around and larger than 1 it accounts for a broad range of non-Gaussian disorder [47]. $G_{0}$ and $\sigma^{2}$ are the first two descriptors of our classification. $G_{0}$ is the geometric mean of the $G_{i}$, also called "typical value", which is for this distribution equal to the median. $\sigma^{2}=\ln \left\{1+\operatorname{Var}\left[G_{i}\right] /\left\langle G_{i}\right\rangle_{P}^{2}\right\}$ is the disorder parameter, related in the indicated way to the relative

\footnotetext{
1 We note that for weak disorder the CPA [47] reduces to the selfconsistent Born approximation, SCBA [41, 44], which is based on Gaussian elastic heterogeneity.
} 
variance. $\Lambda(z)$ is the susceptibility function, which is a linear combination of the longitudinal and transverse local susceptibilities $\chi_{L, T}\left(k_{e}, z\right)$

$$
\begin{aligned}
\Lambda(z) & =\frac{3}{k_{e}^{3}} \int_{0}^{k_{e}} d k k^{4}\left[\frac{4}{3} \frac{1}{-z^{2}+k^{2} v_{L}^{2}(z)}+2 \frac{1}{-z^{2}+k^{2} v_{T}^{2}(z)}\right] \\
& =\frac{4}{3} \chi_{L}\left(k_{e}, z\right)+2 \chi_{T}\left(k_{e}, z\right)
\end{aligned}
$$

The upper wavenumber cutoff $k_{e}$ is the third descriptor of our classification. $k_{e}=\sqrt[3]{2 \pi^{2} / V_{c}}$ is related to the minimum possible coarse-graining volume $V_{c}$ for obtaining statistically independent local shear moduli $G_{i}$ [47]. Therefore $k_{e}$ is inversely proportional to the correlation length $\xi_{G}$ of the fluctuating elastic constants ${ }^{2} G_{i}$. The complex frequency-dependent sound velocities are given by

$$
\rho v_{T}(z)^{2}=G(z) \quad \rho v_{L}(z)^{2}=K_{\exp }+\frac{4}{3} G(z)
$$

Here $\rho=M N / V$ is the mass density, $M$ the molecular mass, $N$ the number of molecules, $V$ the sample volume, and $K_{\exp }$ the experimentally determined bulk modulus. In our model the fluctuations of the local bulk moduli are considered to be negligible [36, 41].

It is easily checked from Eq. (3) that the relation $\chi_{L, T}(k, 0)=v_{L, T}(0)^{-2}$ holds. Therefore in the static $\omega=0$ limit the descriptor $k_{e}$ becomes irrelevant. The zero-frequency limit of the frequency-dependent sound velocities are the experimentally measured sound velocities $v_{T}=v_{T}(0)=\sqrt{G(0) / \rho}=\sqrt{G_{\exp } / \rho}, v_{L}=v_{L}(0)=$ $\sqrt{\left[K_{\exp }+\frac{4}{3} G_{\exp }\right] / \rho}$. These quantities, together with $\rho$ are used as input for our fit procedure for obtaining the material descriptors. The dynamical input are the Raman spectra and the temperature dependent specific heat. Within HET-CPA these vibrational spectra are obtained from the dynamic sound velocities (see Materials and Methods, MM) $v_{L, T}(z)$, which are related to $G(z)$ via relation (4). The latter is calculated self-consistently from the CPA equations (1), (3), and (4) with the condition $G(0)=G_{\text {exp }}$, using the log-normal distribution [2]. The output are the desciptors $G_{0}, \sigma^{2}$ and $k_{e}$. We emphasize that these descriptors have a well-defined physical meaning.

\section{Static descriptors and non-affinity}

Before we discuss the material characterizations obtained by our fit procedure, we now establish a relation between the first two descriptors $G_{0}, \sigma^{2}$ and $G_{\text {exp }}$. As

\footnotetext{
2 Comparing our obtained value of $k_{e}=0.345 \AA^{-1}$ for $\mathrm{SiO}_{2}$ with the correlation length $\xi_{G}=33 \AA$ obtained in a $\mathrm{SiO}_{2}$ simulation [19] we have $k_{e} \xi_{G}=11.4$ as proportionality factor.
}

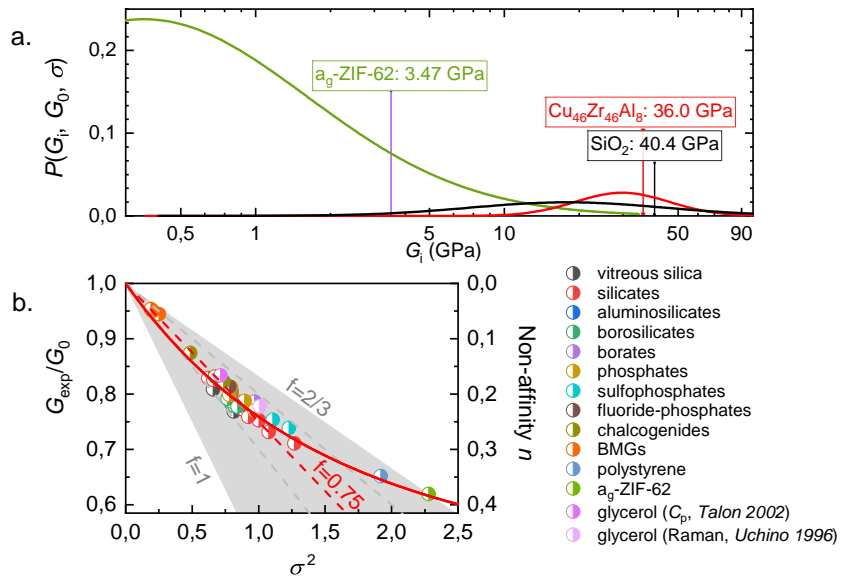

FIG. 1: Panel a: Distribution $P\left(G_{i}, G_{0}, \sigma\right)$ for some archetypal glasses. The labels indicate the characteristic value of $G_{0}$. Panel b: Ratio of the shear modulus $G_{\text {exp }}$ and the typical shear modulus $G_{0}$ vs. the disorder parameter $\sigma^{2}$ (symbols). Curved red line: $G(0) / G_{0}$ vs. $\sigma^{2}$ for $f(\kappa=2)=0.8$ according to the CPA relation (20). Straight dashed lines: Low-disorder CPA relation (6) for several values of $f$. The shaded area indicates the accessible region within the limits of $f(\kappa)$.

shown in MM, the CPA equation (Eq. (20)) for the ratio $G(0) / G_{0}=G_{\exp } / G_{0}$ does not depend further on $G_{0}$, so this ratio is a function of $\sigma^{2}$ with the parameter

$$
f(\kappa)=\frac{1}{3}\left(2+\frac{4}{4+3 \kappa}\right) \quad \kappa=\frac{K_{\text {exp }}}{G_{\text {exp }}}
$$

which varies very slowly with the ratio $\kappa$ between the limits $f(\infty)=2 / 3$ and $f(0)=1$. As further shown in $\mathrm{MM}$, in the small $\sigma^{2}$ limit the relation between $G_{\exp } / G_{0}$ and $\sigma^{2}$ takes the form

$$
\frac{G_{\exp }}{G_{0}}=1-\sigma^{2}\left(f(\kappa)-\frac{1}{2}\right) \equiv 1-n
$$

It has been demonstrated $[41,43]$ that the lowering of the macroscopic shear modulus with respect to the local average is due to non-affine elasticity. Therefore we call $n$ (which increases almost linearly with $\sigma^{2}$ ) the non-affine parameter. Then, relation (6), or its exact counterpart, Eq. (20), combine the parameters $G_{0}$ and $\sigma^{2}$ into a single descriptor, i.e., $\sigma^{2}$ or $n$.

\section{Results}

\section{Non-affinity and ratio $K_{\exp } / G_{\exp }$}

In Fig. 1 we have plotted the ratio $G_{\text {exp }} / G_{0}=1-n$ vs. $\sigma^{2}$ as obtained for a wide range of glassy materials. The full red line corresponds to the exact relation (20) with $f(\kappa)=0.8$, which corresponds to $\kappa=2$, being a representative value for most of our materials. We further see from Fig. 1 that the CPA line for $f=0.8$ closely 


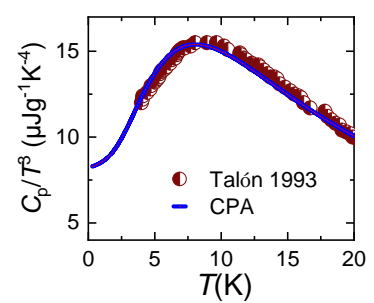

c.

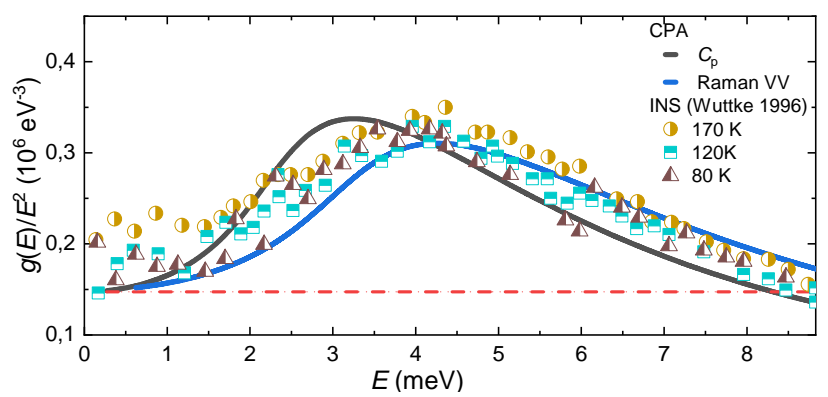

FIG. 2: Example of the CPA fit procedure for vitreous glycerol. The parametric descriptors $G_{0}, \sigma^{2}$ and $k_{e}$ are provided in Supplementary Table S3 [48]. In Panel a the CPA fit is shown for the isobaric heat capacity $C_{p}$ with experimental data extracted from Ref. [49]. Panel b shows the reduced Raman spectra $I_{V V, V H} / \omega[n(\omega)+1]$ extracted from HET-CPA for $\mathrm{VV}$ and $\mathrm{VH}$ geometries, respectively, with experimental data from Ref. [50] (175 K) and using the depolymerization ratio of Kojima [51]. In Panel c the resulting reduced VDoS $g(\omega) / g_{D}(\omega)=g(\omega) \omega_{D}^{3} / 3 \omega^{2}$ are shown. Corresponding experimental VDoS data obtained by inelastic neutron scattering [52] are provided for reference. The dashed red line indicates the Debye level $3 / \omega_{D}^{3}$.

follows the straight dashed line given by equation (6) for $f=0.75$ in the regime $\sigma^{2}<1$, i.e., for low disorder strength.

\section{Spectral properties and the correlation length}

We now turn to the discussion of the influence of disorder on the spectral properties of glasses. We use the term "boson peak" ${ }^{3}$ relating to the prominent maximum of the reduced VDoS $g(\omega) / g_{D}(\omega)$ with $g_{D}(\omega)=3 \omega^{2} / \omega_{D}^{3}$. The Debye frequency is given by $\omega_{D}=k_{D} v_{D}$, where $k_{D}=\sqrt[3]{6 \pi^{2} N / V}$ is the Debye wavenumber and $v_{D}=$ $v_{T}\left[\frac{1}{3}\left(2+\left[\kappa+\frac{4}{3}\right]^{-3 / 2}\right)\right]^{-1 / 3}$ is the Debye velocity. It has been demonstrated in the literature that the boson peak frequency $\omega_{b}$ coincides with the so-called IoffeRegel frequency at which the disorder-induced meanfree path becomes equal to the transverse-acoustic wavelength $[41,42]$. In the frequency range above $\omega_{b}$ the disorder dominates the vibrational spectrum

\footnotetext{
3 Very often in the literature the peak in the reduced Raman spectrum is also called "boson peak", which causes some confusion.
}

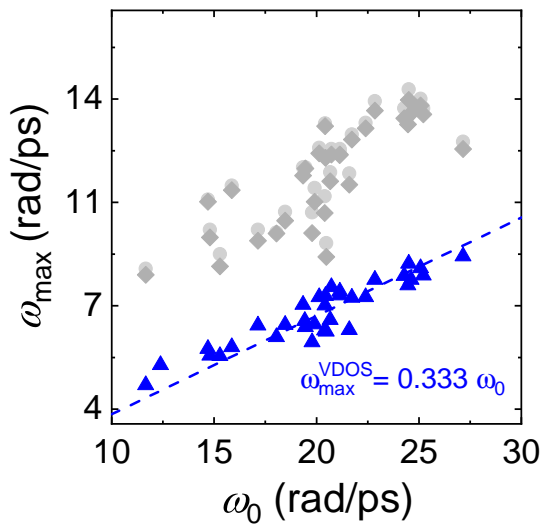

FIG. 3: Frequency positions of the maxima in the reduced Raman intensity (grey diamonds) and in the reduced VDoS (blue triangles) against $\omega_{0}=k_{e} v_{0}$.

In Fig. 2 we demonstrate our fitting procedure for the archetypal example of glycerol. In Panels a and b we plot the the reduced specific heat $C(T) / T^{3}$ and the reduced Raman intensity $I_{V V, V H}(\omega) / \omega[n(\omega)+1]$ (see MM) together with the corresponding CPA fits. It is remarkable that the VDoSs obtained from the two data sets (displayed in Panel c) almost agree to each other. Furthemore, there is a fair agreement with the VDoS data obtained by coherent inelastic neutron scattering [52].

In Fig. 3 we have plotted the boson peak frequency $\omega_{b}$ vs. the characteristic frequency $\omega_{0}=k_{e} v_{0}$, related to the descriptors $k_{e}$ and $G_{0}=\rho v_{0}^{2}$. We see that a precise linear relationship

$$
\omega_{b}=0.333 \omega_{0}
$$

is obtained. Because $\omega_{0} \propto \xi_{G}^{-1}$, we verify that the Ioffe-Regel crossover from wave-like vibrations to disorder dominated ones happens, when the wavelength becomes of the order of the correlation length $\xi_{G}$. In other words, if the mesoscopic transverse sound velocity $v_{0}$ is used to convert from the length scale to the frequency scale $\xi_{G}$ determines the boson peak frequency.

$$
\omega_{b} \propto \omega_{0} \propto \frac{1}{\xi_{G}} \frac{v_{T}}{\sqrt{1-n}} .
$$

In Panel a of Fig. 4 we have plotted the height of the boson peak $\max \left\{g(\omega) / g_{D}(\omega)\right\}$ vs. the relative boson peak position $\omega_{b} / \omega_{D}$. We obtain a power-law relationship

$$
\max \left\{g(\omega) / g_{D}(\omega)\right\}=0.46\left(\frac{\omega_{b}}{\omega_{D}}\right)^{-0.8}=1.11\left(\frac{\omega_{0}}{\omega_{D}}\right)^{-0.8},
$$

where the second equality is obtained from Eq. (7). An even more accurate power-law relationship is displayed in Panel b of Fig. 4 for another dimensionless representation of the boson peak height:

$$
\omega_{0}^{3} \max \left\{g(\omega) / \omega^{2}\right\}=2.19\left(\frac{k_{e}}{k_{D}} \frac{1}{1-n}\right)^{2.42}
$$



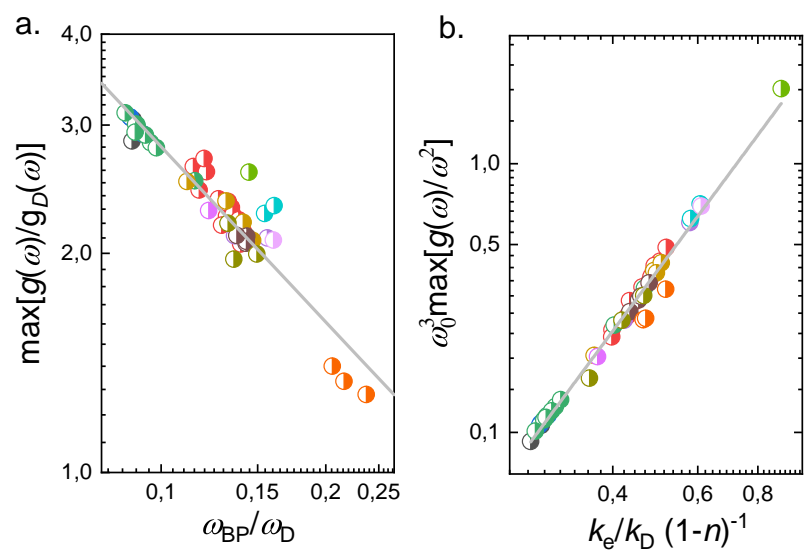

FIG. 4: Panel a: Double-logarithmic plot of the maximum of the reduced VDoS $g(\omega) / g_{D}(\omega)$ vs. $\omega / \omega_{D}$ with $g_{D}(\omega)=$ $3 \omega^{2} / \omega_{D}^{3}$. Panel b: Double-logarithmic plot of the maximum of $\omega_{0}^{3} g(\omega) / \omega^{2}$ vs. $k_{e} / k_{D}(1-n)^{-1}$. The grey lines indicate the exponent 0.8 (panel a) and 2.42 (panel b), respectively.

Relations (7), (9), and (10) relate unambigously the position and the height of the boson peak to the descriptors $k_{e}$ and $G_{0}$

\section{Correlation length, poisson ratio and fragility}

In Fig. 5a we have plotted $k_{e} / k_{D}$ against the Poisson ratio $\nu$ as well as against $\kappa=K_{\exp } / G_{\text {exp }}$, showing that the empirical correlation can be better represented by a linear relation with $\nu$ than by a linear relation with $\kappa$. The correlation with $\nu$ is $\nu=0.06+0.56 k_{e} / k_{D}$.

The Poisson ratio is a rather important figure for designing new glass materials for applications [53, 54]. For example, for $\nu>0.3$ one expects a brittle-to-ductile transition, therefore, values of $k_{e} / k_{D}>0.5$ would point to a ductile material. This makes sense intuitively, as a large value of $\xi_{G} k_{D}$ will lead to a rather rigid network.

A further characteristic property of glasses is the fragility index $m$ of its corresponding liquid [7], which is the relative curvature of the logarithm of the temperature dependence of the viscosity $\eta$ near the glass transition temperature $T_{g}$. It is defined by $m=$ $\partial \log _{10} \eta /\left.\partial\left[T_{g} / T\right]\right|_{T=T_{g}}$. Novikov and Sokolov $[55,56]$ proposed an empirical relationship between the Poisson ratio and $m$ for a number of non-conducting glasses. This had been challenged by Yannopoulos and Johary [57], presenting a large number of exceptions. Later it became clear that such relationships appear only in certain material classes [58]. Very recently Østergaard et al. collected viscosity and elasticity data for a very large number of glasses [54]. They have shown that rather than a correlation a trend exists for larger $m$ values in materials with larger $\nu$ values, see Fig. 6. In Panel $b$ of Fig. 5 we have plotted $m$ against $k_{e} / k_{D}$ of those materials involved in our present study for which viscosity data is vailable in
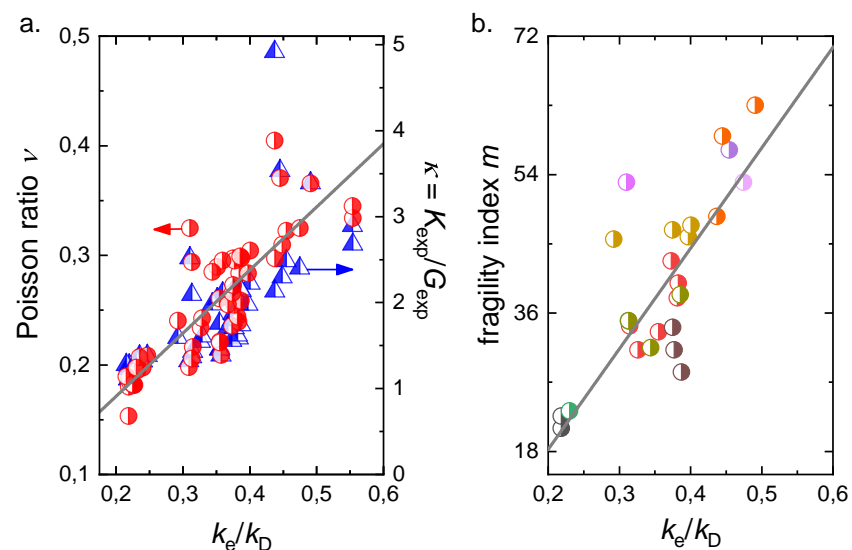

FIG. 5: Panel a: Poisson ratio $\nu$ (red circles, left scale) and $\kappa=K_{\exp } / G_{\exp }$ (blue triangles, right scale) vs. $k_{e} / k_{D}$. Panel b: fragility $m$ vs. $k_{e} / k_{D}$. Symbol code as in Fig. 1 . The grey lines represent linear datafits.

the literature. Indeed we find a weak but notable positive correlation between $m$ and the ratio of $k_{e} / k_{D}$. If we combine this correlation with the one shown in Panel $a$ of Fig. 5 we find $m=218 \nu-17$, which is inserted as red dotted line into the trend picture of Østergaard et al. in Panel b of Fig. 6. We add Sokolov's correlation for non-metals [58] $m=29 \kappa-12$. It is seen that it also fits into Østergaard's trend. For our classification it is important to note that large $k_{e} / k_{D}$ values prevail for fragile materials.

\section{Discussion}

We have already emphasized that our disorder classification essentially reduces to two descriptors, the disorder parameter $\sigma^{2}$, which is proportional to the non-affinity $n=1-G_{\exp } / G_{0}$ and the upper momentum cutoff $k_{e}$, which is inversely proportional to the correlation length $\xi_{G}$ of the fluctuating elastic constant.

The non-affine reduction of the macroscopic shear modulus $G_{\text {exp }}$, which appears in all types of glass, may be interpreted in terms of acoustic percolation [47]. The local sound propagation is fastest in regions of high rigidity and, in turn, the strain energy is localized in the soft, non-affing regions. Under shear, the rigid backbone regions then enclose the non-affine soft regimes, which feature rotational patterns. Metallic glasses with low $\sigma^{2}$ exhibit less pronounced non-affine features. In the more interconnected morphologies, softer modes become a serious obstacle for sound propagation, leading to an enhanced acoustic path length and therefore a reduction of the effective velocity. This is in perfect analogy of the percolational reduction of the conductivity in disordered networks due to disorder [59] and is the reason for the remarkable relation between non-affinity $n$ and $\sigma^{2}$.

The remaining descriptor, the correlation length $\xi_{G}$ of 


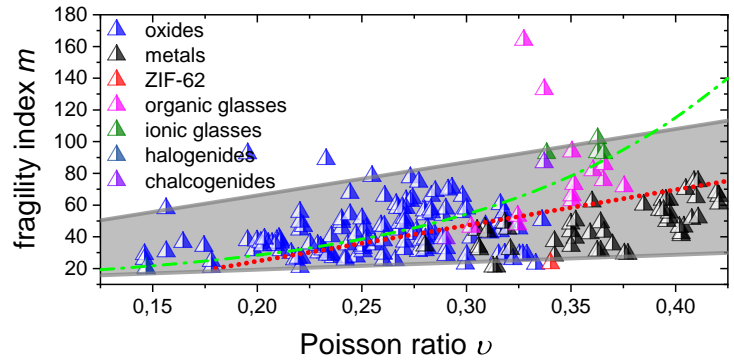

FIG. 6: Symbols: fragility $m$ vs. Poisson ratio $\nu$ as compiled by Østergaard et al. [54]. Dotted red line: Present empirical correlation as obtained from the data of Fig. 5. Dash-dotted green line: Empirical correlation obtained by Sokolov [55, 56, 58] for non-metallic glasses. The grey-shaded region marks the overall trend of reported experimental data.

the spatial rigidity fluctuations features strong correlations with the frequency position $\omega_{b}$ and the height of the boson peak. On the other hand, it is related to the ratio of the shear and bulk modulus $\kappa$, and hence to the Poisson ratio $\nu$ and - trendwise - to the fragility $m$.

Let us start discussing the relation $\omega_{b}=0.333 \omega_{0}$, revealed in Fig. 3. As $\omega_{0}=k_{e} \sqrt{G_{0} / \rho}$ is inversely proportional to $\xi_{G}$, so is the boson peak position. Such a correlation between $\omega_{b}$ and the inverse of a structure-related correlation function was already conjectured in the early days of the discussion of the boson peak [60,61], but had never been unambiguously quantified. The velocity connecting the boson peak frequency and the inverse correlation length turns out to be not related to the macroscopic transverse sound velocity $v_{T}$ but to the average local one, $v_{0}$, i.e., the one related to the typical local shear modulus. Only when a proportionality with $v_{T}$ is enforced, a structure-related pre-factor appears:

$$
\omega_{b} \propto \omega_{0} \propto \frac{1}{\xi_{G}} \frac{v_{T}}{\sqrt{1-n}} .
$$

We see, if $v_{T} / \xi_{G}$ is used instead of $v_{0} / \xi_{G}$, a correction factor $[1-n]^{-1 / 2}$ appears, which is similar to an empirical "shape factor", introduced by Duval et al. [61] for accounting for specific material properties in order to improve the correlation between $\omega_{b}$ and $v_{T} / \xi_{G}$. Instead of the shape of rigid inclusions [61], our correction factor accounts for the non-affinity and, by the CPA equivalence (6), to the disorder strength.

Summarizing the trends displayed in Fig. 6, and combining the findings of Figs. 5 and 1, we may say that with increasing liquid fragility, the correlation length (relative to the intermolecular distance) decreases and nonaffinity (weakly) increases. The former observation is a strong confirmation for the correlation between liquid heterogeneity and heterogeneity in the solid (glassy) state which was previously found for molecular glass formers and colloidal suspensions $[62,63]$, where the growing size of correlated regions was identified as the origin of the dynamic slow-down during glass formation (further support may be found in the correlation between liquid fragility and structural fragility which was discovered for metallic glasses [64]). It is, indeed, known that structures presenting an abundance of soft elastic modes tend to have a higher length scale of shear fluctuation $\xi_{G}$ and are strong (low Poisson's ratio). Previous modelling studies [65] found that the amplitude of the boson peak (in Debye units) correlates well with the inverse of the fragility, $1 / \mathrm{m}$. Our data provide experimental confirmation for this observation, as relation (9) implies $\max \left\{g(\omega) / g_{D}(\omega)\right\} \propto\left(k_{e} / k_{D}\right)^{-0.8} \propto m^{-0.8}$.

The metal-organic framework glass represents a particular case. A glass formed from ZIF-62, termed $\mathrm{a}_{g^{-}}$ ZIF-62, was obtained through structural collapse of a nanoporous crystal at temperatures only mildly above $T_{m}$. As a relic of the crystalline state, the resulting glass contains pores on the scale of $0.5 \mathrm{~nm}$ [66], which represents a disperse morphology of soft modes. In terms of the governing length scales and the function of $f(\kappa)$, their properties are similar to metallic glasses. However, among all the considered glass chemistries, they exhibit the highest non-affinity and the highest disorder parameter.

\section{Conclusion}

In conclusion, we implemented heterogeneouselasticity theory in conjunction with the coherentpotential approximation using experimental input from a broad variety of glass chemistries in order to extract the vibrational density of states and the probability distribution density of the local shear modulus of glassy materials. The exclusive use of physically meaningful fitting parameters enables widespread application of our approach to real-world materials, revealing remarkable trends which appear to hold across all classes of glass (despite pronounced differences in bond directionality and localization).

In analyzing our data, we extracted three universal descriptors of disorder and the statistics of elastic heterogeneity: The typical value $G_{0}$ and the disorder parameter $\sigma^{2}$, which characterize the log-normal distribution function, and the momentum cutoff $k_{e}$, which is inversely proportional to the correlation length scale $\xi_{G}$. Using experimental information on the macroscopic shear modulus $G_{\exp }$, we find a one-to-one relation between the nonaffinity $n=1-G_{\text {exp }} / G_{0}$ and $\sigma^{2}$. This leaves only two independent descriptors, the disorder parameter $\sigma^{2}$ and the cutoff wavenumber $k_{e}$, viz. the correlation length. We found a linear one-to-one correlation between the frequency $\omega_{0} \propto k_{e} \sqrt{G_{0}}$ and the boson peak position $\omega_{b}$ and a power-law relation between the boson peak amplitude and frequency positions in Debye units. We further find a correlation between $k_{e}$ and the Poisson ratio and the fragility.

It remains to be explored how disordered materials 
derived from techniques other than liquid quenching (e.g., the prolific superstrong glasses [67] or pressureamorphized crystals [66]) would fall into the observed trends. Furthermore, extending the range of observation to materials at the extremes of disorder and with enhanced non-affinity, respectively, would be a significant step in understanding the further role of structural heterogeneity on macroscopic properties through refining the discovered relations, for example, by considering pressurized or autoclaved glasses and mesoporous hybrids beyond the very few examples which are known today. Such insight will open new routes for specific and independent tailoring of non-affinity and structural heterogeneity towards macroscopic properties. Aside mechanical and vibrational behavior, this concerns also transport coefficients sound propagation, heat and ion conduction.

\section{Ackowledgements}

This project has received funding from the European Research Council (ERC) under the European Union's Horizon 2020 research and innovation program (L.W., ERC grant UTOPES, grant agreement no. 681652). T.D.B acknowledges the Royal Society for a University Research Fellowship (UF150021) and the Leverhulme Trust for a Philip Leverhulme Prize.

\section{Materials and methods}

\section{Materials}

The glass samples used in this study were chosen on the condition of individual material homogeneity and availability of general physical data so as to cover a broad range of materials with variable bonding character and variations in fundamental structural motifs and network dimensionality. All studied compositions are summarized in Table S1 [48], together with their glass transition temperature $T_{g}$, liquid fragility index $m$, mass density $\rho$ and calculated values of the volume density of bond energy $<U_{0} / V_{0}>$ and of the atomic packing density $C_{g}$. The experimental (macroscopic) shear, bulk and Young's modulus $G_{e x p}, K_{e x p}$ and $E_{e x p}$, respectively and Poisson's ratio $\nu$ are given in Table S2 [48]. Most glasses were prepared by conventional melt-quenching techniques. Some commercial samples were used as obtained, including a standard soda-lime silicate glass (Marienfeld Superior, Paul Marienfeld, Lauda-Königshofen, Germany), a lowalkali borosilicate glass (Borofloat-33, Schott TGS, Jena, Germany), two variants of vitreous silica (Suprasil, Heraeus Quarzglas, Hanau, Germany, and R300, prepared by a modified chemical vapor deposition route (MCVD), respectively) and a range of optical glasses (Schott, Mainz, Germany). Detailed information on the fabrication procedures are provided in the References given in Tables S12 [48]. All materials were used in the form of disks with a thickness of 1-5 $\mathrm{mm}$ and a diameter of $2 \mathrm{~mm}$, polished to optical grade using $\mathrm{CeO}_{2}$ suspensions. X-ray diffraction (MiniFlex600, Rigaku) was conducted on all samples in order to confirm the absence of any detectable crystalline species. The glass transition temperatures were determined by differential scanning calorimetry (Netzsch STA 449 F3 Jupiter) in flowing nitrogen, employing heating rates of $20 \mathrm{~K} / \mathrm{min}$ for the MeP, SP and $\mathrm{a}_{g}$-ZIF-62 glasses, $40 \mathrm{~K} / \mathrm{min}$ for $\mathrm{Cu}_{46} \mathrm{Zr}_{46} \mathrm{Al}_{8}(40 \mathrm{~K} / \mathrm{min})$ and 10 $\mathrm{K} / \mathrm{min}$ for all other glasses. Density data were obtained with an Archimedes balance using distilled water or dry ethanol as immersion media. Where not already available from previous studies (for References, see Table S2 [48]), the macroscopic (effective) elastic properties were analyzed through ultrasonic echography at room temperature. The longitudinal and transversal sound velocities $v_{L}$ and $v_{T}$ were derived from the corresponding sound wave propagation times (recorded with an accuracy of \pm $1 \mathrm{~ns}$ by piezoelectric transducers operating at frequencies of 8 to $12 \mathrm{MHz}$ ) and the exact thickness of the polished glass plates (determined with an accuracy of $\pm 2 \mu \mathrm{m}$ using a micrometer screw). The macroscopic elastic moduli were calculated from these values.

\section{Vibrational spectra}

Raman scattering spectroscopy was carried-out using a Renishaw inVia confocal Raman microscope equipped with a low-frequency notch filter covering the frequencyrange of $0-200 \mathrm{~cm}^{-1}$. Samples were excited with an Argon ion laser operating at $514.5 \mathrm{~nm}$. The signal was collected with a CCD camera at a spectral resolution of $2 \mathrm{~cm}^{-1}$, using a grating with 2400 lines $/ \mathrm{mm}$ and a 50x microscope objective. All spectra were collected in two polarization geometries, $\mathrm{VV}$ and $\mathrm{VH}$, by means of a polarizer/half-wave plate set-up inserted in the laser beamline between the notch filter and the monochromator. For the chalcogenides and the metallic glasses, the vibrational properties were investigated by heat capacity $\left(C_{p}\right)$ measurements at low temperature. For the chalcogenides, this was a precautious measure because the relatively harsh Raman excitation conditions might have induced experimental artifacts, which would compromise subsequent data analyses. For the metallic glasses, they are generally unsuitable for Raman spectroscopy because first-order Raman bands result from a change in the polarizability of the molecule and metallic materials are infinitely polarizable. Further low-temperature heat capacity data on vitreous silica and on the aluminosilicate glasses (AS) were extracted from a previous study [68]. $C_{p}$ analyses of the chalcogenide glasses $\mathrm{As}_{38} \mathrm{Se}_{62}$ and $\mathrm{GeSe}_{4}$, and of the metallic glasses $\mathrm{Pd}_{40} \mathrm{Ni}_{40} \mathrm{P}_{20}$ and $\mathrm{Zr}_{52.5} \mathrm{Cu}_{17.9} \mathrm{Ni}_{14.6} \mathrm{Al}_{10} \mathrm{Ti}_{5}$ were conducted on a physical property measurement system (PPMS, Quantum Design) at temperatures down to approximately $2.3 \mathrm{~K}$. $C_{p}$ data on vitreous $\mathrm{As}_{2} \mathrm{~S}_{3}$ [69] and $\mathrm{Cu}_{46} \mathrm{Zr}_{46} \mathrm{Al}_{8}$ [70] were taken from literature, as were all experimental data of glycerol 
[49-52].

\section{CPA calculation of spectral functions}

From the CPA equations (1), (3), and (4) the dynamic sound velocities $v_{L, T}(z)$ are obtained via $G(z)$. From these quantities the VDoS is obtained as

$g(\omega)=\frac{2 \omega}{3 \pi} \frac{3}{k_{D}^{3}} \int_{0}^{k_{D}} d k k^{2}\left[\frac{1}{-z^{2}+k^{2} v_{L}^{2}(z)}+2 \frac{1}{-z^{2}+k^{2} v_{T}^{2}(z)}\right]$

Here $k_{D}=\sqrt[3]{6 \pi^{2} N / V}$ is the Debye wavenumber. The temperature dependent specific heat is given by

$$
C_{p}(T)=A_{c} \int_{0}^{\infty} g(\omega) \frac{\omega^{2}}{T^{2}} \frac{e^{\frac{\hbar \omega}{k_{B} T}}}{\left(e^{\frac{\hbar \omega}{k_{B} T}}-1\right)^{2}} d \omega
$$

$A_{c}$ is a prefactor and $\hbar$ and $k_{B}$ are Planck's and Boltzmann's constants. The depolarized $(V H)$ and polarized $(V V)$ Raman spectra are $[31,36,46]$

$I_{V H}(\omega)=A_{V H}[n(\omega)+1]\left(\frac{4}{3}\left[\chi_{L}\left(k_{a}, \omega\right)\right]^{\prime \prime}+2\left[\chi_{T}\left(k_{a}, \omega\right)\right]^{\prime \prime}\right)$

$$
I_{V V}(\omega)=A_{V V}[n(\omega)+1]\left[\chi_{L}\left(k_{a}, \omega\right)\right]^{\prime \prime}+\frac{4}{3} I_{V H}(\omega)
$$

$A_{V H}$, and $A_{V V}$ are prefactors and $k_{a}$ is a cutoff related to the correlation length of the Pockels-constant fluctuations $n(\omega)=\left[e^{\hbar \omega / k_{B} T}-1\right]^{-1}$ is the boson occupation number (to which the boson peak owes its name). In addition to $\sigma^{2}, G_{0}, k_{e}$ the Raman spectra feature two further descriptors ("Raman descriptors"), which are the wavenumber cutoff $k_{a} \propto \xi_{a}^{-1}$, where $\xi_{a}$ is the correlation length of the pockels-constant fluctuations and the amplitude ratio $A_{V V} / A_{V H}$ of the two types of Pockelsconstant correlation functions [31]. As in previous studies $[31,33,46], k_{a}$ turned out to be very near to $k_{e}$, see Table S3 [48].

\section{CPA equation for $\omega=0$}

The CPA equation for $\omega=0$ is

$$
\begin{aligned}
0 & =\left\langle\frac{G_{i}-G(0)}{1+\frac{1}{3}\left[G_{i}-G(0)\right] \Lambda(0)}\right\rangle_{P} \\
\Leftrightarrow \quad G(0) & =\left\langle\frac{G_{i}}{1+\frac{1}{3}\left[G_{i}-G(0)\right] \Lambda(0)}\right\rangle_{P}
\end{aligned}
$$

We have

$$
\frac{1}{3} \Lambda(0)=\frac{1}{3}\left(\frac{2}{G_{\exp }}+\frac{4}{3} \frac{1}{K_{\exp }+\frac{4}{3} G_{\exp }}\right)=f(\kappa) \frac{1}{G_{\exp }}
$$

with

$$
f(\kappa)=\frac{1}{3}\left(2+\frac{4}{4+3 \kappa}\right) \quad \kappa=\frac{K_{\text {exp }}}{G_{\text {exp }}}
$$

We note that $f(\kappa)$ is a very slowly varying function with

$$
f(\infty)=\frac{2}{3} \leq f(\kappa) \leq f(0)=1
$$

We now introduce dimensionless variables into the second version of Eq. (16): $g_{i}=G_{i} / G_{0}$ and $Q=G_{\exp } / G_{0}$ and obtain

$$
Q=\frac{1}{\sqrt{2 \pi} \sigma} \int_{0}^{\infty} d g_{i} \frac{e^{-\frac{1}{2 \sigma^{2}} \ln \left\{g_{i}^{2}\right\}}}{1+f(\kappa)\left[\frac{g_{i}}{Q}-1\right]}
$$

We see that $Q$ is an implicit function of $\sigma^{2}$ (and vice versa) with $f(\kappa)$ as parameter

\section{CPA calculation of $G_{\exp }$ in the small-disorder limit}

We now define new deviatoric moduli:

$$
G_{\text {exp }}=\left\langle G_{i}\right\rangle_{P}-\Sigma \quad G_{i}=\left\langle G_{i}\right\rangle_{P}-\Delta_{i}
$$

The CPA equation (16) becomes

$0=\left\langle\frac{\Delta_{i}-\Sigma}{1-\frac{f(\kappa)}{G_{\exp }}\left[\Delta_{i}-\Sigma\right]}\right\rangle_{P} \Leftrightarrow \Sigma=\left\langle\frac{\Delta_{i}}{1-\frac{f(\kappa)}{G_{\exp }}\left[\Delta_{i}-\Sigma\right]}\right\rangle_{P}$

We now treat $\Delta_{i}$ and $\Sigma$ to be of order $\sigma$ and obtain to lowest (quadratic) order

$$
\Sigma \approx \frac{f(\kappa)}{\left\langle G_{i}\right\rangle_{P}}\left\langle\Delta_{i}^{2}\right\rangle_{P}
$$

Therefore

$$
\frac{G_{\exp }}{\left\langle G_{i}\right\rangle_{P}}=1-\frac{\Sigma}{\left\langle G_{i}\right\rangle_{P}} \approx 1-f(\kappa) \frac{\left\langle\Delta_{i}^{2}\right\rangle_{P}}{\left\langle G_{i}\right\rangle_{P}^{2}}=1-f(\kappa)\left(e^{\sigma^{2}}-1\right)
$$

and finally

$$
\begin{aligned}
Q & =\frac{G_{\text {exp }}}{G_{0}}=\frac{G(0)}{\left\langle G_{i}\right\rangle_{\text {geo }}}=e^{\frac{1}{2} \sigma^{2}}\left[1-f(\kappa)\left(e^{\sigma^{2}}-1\right)\right] \\
& \approx 1-\left(f(\kappa)-\frac{1}{2}\right) \sigma^{2}
\end{aligned}
$$

\section{Numerical implementation}

The CPA equation can be solved numerically within a few iterations. The resulting solution, the frequencydependent shear modulus $G(z)$, was then used to obtain the vibrational density of states $g(\omega)$. Fits were constructed in terms of a least square minimization of the difference between the experimental data 
and the corresponding theoretical predictions of Eq. (13-15). There were, therefore, two independent fitting procedures involved in this work: the $C_{p}$-fit and the Raman-fit. The $C_{p}$-fit produced $\left(\sigma, G_{0}, k_{e}\right)$ while taking $\left(v_{L}, v_{T}, k_{D}, C_{p}(2.3<T<20 K)\right)$ for input. The Raman-fit produced $\left(\sigma, G_{0}, k_{e}, k_{a}, A_{21}\right)$ by using $\left(v_{L}, v_{T}, k_{D}, I_{V V}(\omega), I_{V H}(\omega)\right)$ for input, where $A_{21}=$ $A_{V H} / A_{V V}$. The frequency ranges for Raman spectra were selected from $15 \mathrm{~cm}^{-1}$ below the Raman boson peak up to $100 \mathrm{~cm}^{-1}$. Since the fitting problems are ill-posed, we employed Tikhonov regularization. As the criterion for identifying the optimized regularization parameter for the $C_{p}$-fit we used the minimum of the smoothness parameter $M$ of the reduced $\operatorname{VDoS}$ ( $M$ increases sharply if the reduced VDoS starts to oscillate). In this way, an independent, individually optimized regularization parameter was attributed to each glass dataset. For the Ramanfit, the goodness of fit $G O F$ was additionally used. GOF evaluates the maximum curve length of an experimental Raman spectrum which fits to the predicted one. It is noteworthy that, although we restricted the Raman fit input to the spectral range of $<100 \mathrm{~cm}^{-1}$, the obtained fits of most samples showed good agreement to experimental data also up to higher frequency $(<200$ $\left.\mathrm{cm}^{-1}\right)$. The full evaluation procedure was scripted in order to produce independent and automated fits for Raman and $C_{p}$ experimental data, respectively. In this way, the VDoS was derived independently from two different experimental datasets.

\section{Competing interests}

The authors declare no competing interests.

\section{Author contributions}

LW conceived of the experiments and wrote the manuscript together with WS. WS, ZP and LW developed the extended HET-CPA. ZP was responsible for numerical implementation and data fits. OB was responsible for the Raman spectroscopic analysis. OB, SS, AK, RL, TDB and GW provided sample material and acquired the supporting physical data, including lowtemperature heat capacity measurements. All authors participated in data analysis and evaluation, and were involved in the discussion and manuscript revisions.
[1] W. H. Zachariasen, Journal of the American Chemical Society 54, 3841 (1932).

[2] D. Turnbull and M. H. Cohen, The Journal of chemical physics 34, 120 (1961).

[3] Y. Cheng and E. Ma, Progress in materials science 56, 379 (2011).

[4] G. Biroli and J. P. Garrahan, J. Chem. Phys. 138, 12A301 (2013).

[5] L. Berthier and G. Biroli, Rev. Mod. Phys. 83, 587 (2011).

[6] M. D. Ediger, Annual review of physical chemistry 51, 99 (2000).

[7] C. A. Angell, Science 267, 1924 (1995).

[8] B. Ruta, G. Monaco, V. Giordano, F. Scarponi, D. Fioretto, G. Ruocco, K. Andrikopoulos, and S. Yannopoulos, The Journal of Physical Chemistry B 115, 14052 (2011).

[9] A. Makishima and J. D. Mackenzie, Journal of Noncrystalline solids 17, 147 (1975).

[10] Y. Shi, A. Tandia, B. Deng, S. R. Elliott, and M. Bauchy, Acta Materialia (2020).

[11] E. D. Cubuk, R. Ivancic, S. S. Schoenholz, D. Strickland, A. Basu, Z. Davidson, J. Fontaine, J. L. Hor, Y.-R. Huang, Y. Jiang, et al., Science 358, 1033 (2017).

[12] A. Nicolas, E. E. Ferrero, K. Martens, and J.-L. Barrat, Reviews of Modern Physics 90, 045006 (2018).

[13] B. Wang, Y. Yu, M. Wang, J. C. Mauro, and M. Bauchy, Physical Review B 93, 064202 (2016).

[14] O. Benzine, S. Bruns, Z. Pan, K. Durst, and L. Wondraczek, Advanced Science 5, 1800916 (2018).

[15] E. J. Frankberg, J. Kalikka, F. G. Ferré, L. Joly-Pottuz, T. Salminen, J. Hintikka, M. Hokka, S. Koneti, T. Douil- lard, B. Le Saint, et al., Science 366, 864 (2019).

[16] J. P. Wittmer, A. Tanguy, J.-L. Barrat, and L. Lewis, Europhys. Lett. 57, 423 (2002).

[17] B. DiDonna and T. Lubensky, Physical Review E 72, 066619 (2005)

[18] F. Leonforte, R. Boissière, A. Tanguy, J. Wittmer, and J.-L. Barrat, Physical Review B 72, 224206 (2005).

[19] F. Leonforte, A. Tanguy, J. Wittmer, and J.-L. Barrat, Physical review letters 97, 055501 (2006).

[20] M. Tsamados, A. Tanguy, C. Goldenberg, and J.-L. Barrat, Phys. Rev. B 80, 026112 (2009).

[21] J. Ding, Y.-Q. Cheng, H. Sheng, M. Asta, R. O. Ritchie, and E. Ma, Nature communications 7, 1 (2016).

[22] W. A. Phillips and A. Anderson, Amorphous solids: lowtemperature properties, vol. 24 (Springer, 1981).

[23] U. Buchenau, N. Nücker, and A. Dianoux, Phys. Rev. Lett. 53, 2316 (1984).

[24] B. Frick and D. Richter, Science 267, 1939 (1995).

[25] A. I. Chumakov, Sergueev, U. van Bürck, W. Schirmacher, T. Asthalter, R. Rüffer, O. Leupold, and W. Petry, Phys. Rev. Lett. 92, 245508 (2004).

[26] J. Wuttke, W. Petry, C. Coddens, and F. Fujara, Phys. Rev. E 52, 4026 (1995).

[27] G. Monaco and V. M. Giordano, PNAS 106, 3659 (2009).

[28] G. Baldi, A. Fontana, G. Monaco, L. Orsingher, S. Rols, F. Rossi, and B. Ruta, Phys. Rev. Lett. 102, 195502 (2009).

[29] G. Baldi, V. M. Giordano, G. Monaco, and B. Ruta, Phys. Rev. Lett. 104, 195501 (2010).

[30] G. Baldi, V. M. Giordano, and G. Monaco, Phys. Rev. B 83, 174203 (2011)

[31] B. Schmid and W. Schirmacher, Phys. Rev. Lett. 100, 
137402 (2008), see references therein for an extended list of Raman-spectroscopy papers.

[32] S. Caponi, S. Corezzi, D. Fioretto, A. Fontana, G. Monaco, and F. Rossi, Phys. Rev. Lett. 102, 027402 (2009).

[33] A. Schulte, W. Schirmacher, B. Schmid, and T. Unruh, J. Phys. Condens. Matter 23, 254212 (2011).

[34] M. A. Ramos, C. Talón, and S.Vieira, J. Non-Cryst. Solids 307 - 310, 80 (2002).

[35] M. A. Ramos, Low-temperature Phys. 104, 46 (2020).

[36] W. Schirmacher, T. Scopigno, and G. Ruocco, Journal of Non-Crystalline Solids 407, 133 (2015).

[37] T. Nakayama, Rep. Prog. Phys. 65, 1195 (2002).

[38] M. I. Klinger, Phys. Rep. 292, 111 (2010).

[39] A. I. Chumakov et al., Phys. Rev. Lett. 106, 225501 (2011).

[40] A. I. Chumakov et al., Phys. Rev. Lett. 112, 025502 (2014).

[41] A. Marruzzo, W. Schirmacher, A. Fratalocchi, and G. Ruocco, Scientific reports 3, 1407 (2013).

[42] H. Shintani and H. Tanaka, Nature materials 7, 870 (2008).

[43] H. Mizuno, S. Mossa, and J.-L. Barrat, Physical Review E 87, 042306 (2013).

[44] W. Schirmacher, EPL (Europhys. Lett.) 73, 892 (2006).

[45] W. Schirmacher, G. Ruocco, and T. Scopigno, Phys. Rev. Lett. 98, 025501 (2007).

[46] B. Schmid, Theory of low-frequency raman-scattering and other vibrational properties of disordered solids (2007), diploma thesis.

[47] S. Köhler, G. Ruocco, and W. Schirmacher, Physical Review B 88, 064203 (2013).

[48] See Supplementary Information.

[49] C. Talón, Q. W. Zou, M. A. Ramos, R. Villar, and S. Vieira, Phys. Rev. B 65, 012203 (2002).

[50] T. Uchino and T. Yoko, Science 273, 480 (1996).

[51] S. Kojima, Phys. Rev. B 47, 2924(R) (1993).

[52] J. Wuttke, W. Petry, and S. Pouget, The Journal of Chemical Physics 105, 5177 (1996).

[53] G. N. Greaves, A. L. Greer, R. S. Lakes, and T. Rouxel, Nature Materials 10, 823 (2011).
[54] M. B. Østergaard, S. R. Hansen, K. Januchta, T. To, S. J. Rzoska, M. Bockowski, M. Bauchy, and M. M. Smedskjaer, Materials 12, 2439 (2019).

[55] M. N. Novikov and A. P. Sokolov, Nature 431, 961 (2004).

[56] M. N. Novikov, Y. Ding, and A. P. Sokolov, Phys. Rev. E 71, 061501 (2005).

[57] S. N. Yannopoulos and G. P. Johari, Nature 442, E7 (2006).

[58] M. N. Novikov and A. P. Sokolov, Phys. Rev. B 74, 064203 (2006).

[59] A. I. Efros and B. I. Shklovskii, Electronic properties of doped semiconductors (Springer-Verlag, Heidelberg, 1984).

[60] S. Elliott, EPL (Europhys. Lett.) 19, 201 (1992).

[61] E. Duval, A. Boukenter, and T. Achibat, Journal of Physics: Condensed Matter 2, 10227 (1990).

[62] X. Qiu and M. Ediger, The Journal of Physical Chemistry B 107, 459 (2003).

[63] L. Berthier, G. Biroli, J.-P. Bouchaud, L. Cipelletti, D. El Masri, D. L'Hôte, F. Ladieu, and M. Pierno, Science 310, 1797 (2005).

[64] N. Mauro, M. Blodgett, M. Johnson, A. Vogt, and K. Kelton, Nature communications 5, 1 (2014).

[65] L. Yan, G. Düring, and M. Wyart, Proceedings of the National Academy of Sciences 110, 6307 (2013).

[66] R. N. Widmer, G. I. Lampronti, S. Anzellini, R. Gaillac, S. Farsang, C. Zhou, A. M. Belenguer, C. W. Wilson, H. Palmer, A. K. Kleppe, et al., Nature materials 18, 370 (2019).

[67] S. Singh, M. D. Ediger, and J. J. De Pablo, Nature materials 12, 139 (2013).

[68] M. F. Ando, O. Benzine, Z. Pan, J.-L. Garden, K. Wondraczek, S. Grimm, K. Schuster, and L. Wondraczek, Sci. Rep. 8, 5394 (2018).

[69] B. Terziyska, I. Bivas, and K. Nenkov, Cryogenics 49, 171 (2009).

[70] Y. Li, P. Yu, and H. Bai, Journal of Applied Physics 104, 013520 (2008) 